\title{
Advanced trauma life support training for ambulance crews (Review)
}

\author{
Jayaraman S, Sethi D
}

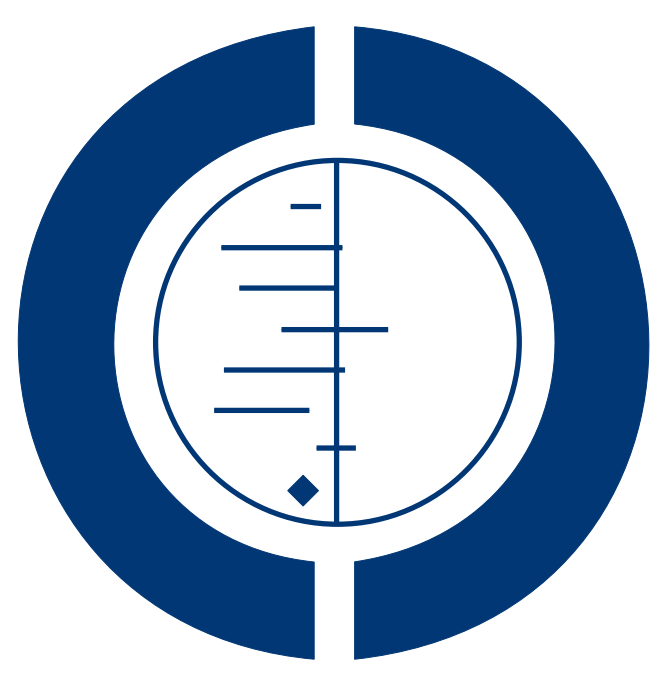

\section{THE COCHRANE COLLABORATION $^{\circledR}$}

This is a reprint of a Cochrane review, prepared and maintained by The Cochrane Collaboration and published in The Cochrane Library 2010, Issue 1

http://www.thecochranelibrary.com

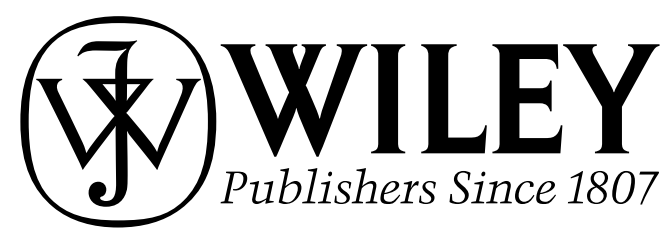

Advanced trauma life support training for ambulance crews (Review)

Copyright $(2010$ The Cochrane Collaboration. Published by John Wiley \& Sons, Ltd. 
TABLE OF CONTENTS

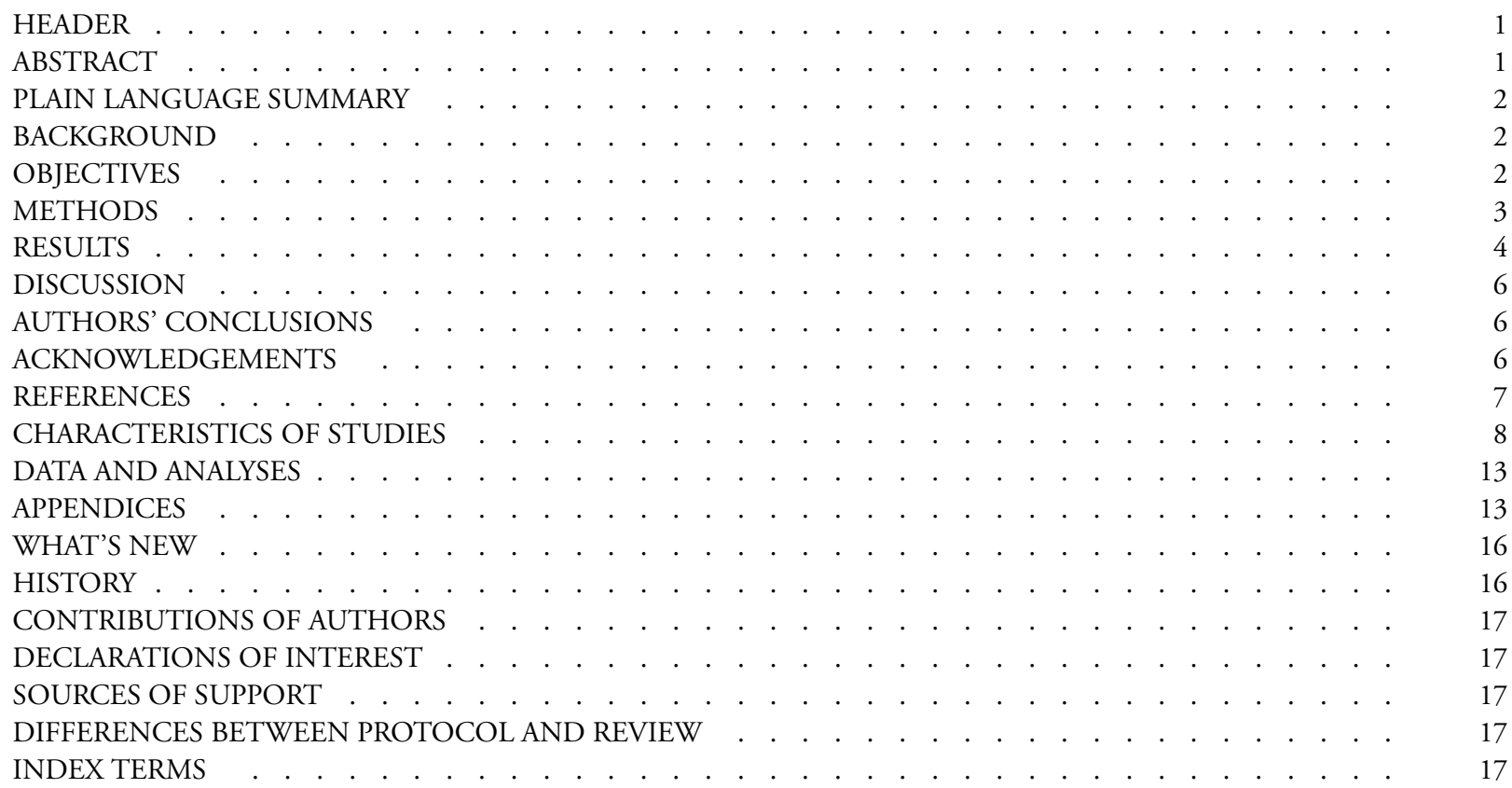

Advanced trauma life support training for ambulance crews (Review)

Copyright (C) 2010 The Cochrane Collaboration. Published by John Wiley \& Sons, Ltd. 


\title{
[Intervention Review]
}

\section{Advanced trauma life support training for ambulance crews}

\author{
Sudha Jayaraman ${ }^{2}$, Dinesh Sethi ${ }^{1}$ \\ ${ }^{1}$ WHO European Centre for Environment and Health, Rome 00187, Italy. ${ }^{2}$ Department of Surgery, University of California San \\ Francisco, San Francisco, CA, USA \\ Contact address: Dinesh Sethi, WHO European Centre for Environment and Health, Via F Crispi 10, Rome 00187, Italy. \\ din@ecr.euro.who.int.
}

Editorial group: Cochrane Injuries Group.

Publication status and date: Edited (conclusions changed), published in Issue 1, 2010.

Review content assessed as up-to-date: 7 July 2009.

Citation: Jayaraman S, Sethi D. Advanced trauma life support training for ambulance crews. Cochrane Database of Systematic Reviews 2010, Issue 1. Art. No.: CD003109. DOI: 10.1002/14651858.CD003109.pub2.

Copyright (c) 2010 The Cochrane Collaboration. Published by John Wiley \& Sons, Ltd.

\section{A B S T R A C T}

\section{Background}

There is an increasing global burden of injury especially in low- and middle-income countries (LMICs). To address this, models of trauma care initially developed in high income countries are being adopted in LMIC settings. In particular, ambulance crews with advanced life support (ALS) training are being promoted in LMICs as a strategy for improving outcomes for victims of trauma. However, there is controversy as to the effectiveness of this health service intervention and the evidence has yet to be rigorously appraised.

\section{Objectives}

To quantify the impact of ALS-trained ambulance crews versus crews without ALS training on reducing mortality and morbidity in trauma patients.

\section{Search strategy}

Searches were not restricted by date, language or publication status. We searched the Cochrane Injuries Group Specialised Register, CENTRAL (The Cochrane Library 2009, Issue 3), MEDLINE (Ovid SP), EMBASE (Ovid SP), CINAHL (EBSCO) and PubMed in all years up to July 2009. We also searched the reference lists of relevant studies and reviews in order to identify unpublished material.

\section{Selection criteria}

Randomised controlled trials, quasi-randomised controlled trials and non-randomised studies, including before-and-after studies and interrupted time series studies, comparing the impact of ALS-trained ambulance crews versus crews without ALS training on the reduction of mortality and morbidity in trauma patients.

\section{Data collection and analysis}

One review author applied eligibility criteria to trial reports for inclusion and extracted data.

\section{Main results}

We found one controlled before-and-after trial, one uncontrolled before-and-after study, and one randomised controlled trial that met the inclusion criteria. None demonstrated evidence to support ALS training for pre-hospital personnel. In the uncontrolled beforeand-after study, 'a priori' sub-group analysis showed an increase in mortality among patients who had a Glasgow Coma Scale score of less than nine and received care from ALS trained ambulance crews. Additionally, when the pre-hospital trauma score was taken into account in logistic regression analysis, mortality in the patients receiving care from ALS trained crews increased significantly. 


\section{Authors' conclusions}

At this time, the evidence indicates that there is no benefit of advanced life support training for ambulance crews.

\section{PLAIN LANGUAGE SUMMARY}

No evidence to suggest that advanced life support training for ambulance crews cuts death rates or decreases disability in injured people

Injury is one of the top ten causes of death and disability worldwide. It results in an early loss of life for many young people and ongoing high medical care costs. Advanced life support (ALS) training for ambulance crews is believed to have contributed to a reduction in the number of deaths from injury in predominantly high-income countries where this service is available. ALS services are also being adapted for low- and middle-income countries. This review of trials found there is no evidence to suggest that ALS training for ambulance personnel improves the outcomes for injured people.

\section{B A C K G R O U N D}

The epidemiological, demographic, and socio-political transitions underway in many countries are associated with an increasing burden of disease from injury, particularly in low- and middleincome countries (LMICs). These findings have been highlighted by the Global Burden of Disease Study, which identified injury as one of the top ten causes of death and disability worldwide (Murray 1997a; Murray 1997b; Murray 1997c; Lopez 2006). That study also predicted that the incidence of injury was likely to increase by the year 2030 (Mathers 2006). Although infectious diseases are still extremely important causes of death in LMICs, the challenges of injury and non-communicable disease add to these as important causes of premature mortality and morbidity (Gwatkin 1997). Injuries place a disproportionately large burden of disease on young people (Murray 1997a; Murray 1997b), and, consequently, are a leading cause of premature loss of productive life, of high medical care costs, of significant degrees of disability and of large socio-economic loss to society (Berger 1996).

There have been recent calls by the public health community and civil organisations to formulate a strategy to decrease the burden from injuries. While responding to injuries requires considerable attention to preventive efforts (Berger 1996), improvements in health care provision which reduce deaths, disability and societal costs are also required (Sethi 2000). In many high income countries (HICs), reductions in trauma mortality of $15 \%$ to $20 \%$ have been achieved in the last few decades (Cales 1984; Roberts 1996; Lecky 2000), which may be partly as a result of improved systems for trauma care. Advanced life support (ALS) training for ambulance personnel is considered to have made an important contribution to the reduction of trauma mortality in HIC settings (Kirsch 1998; Reines 1998). ALS-trained ambulance crews receive extra training in endotracheal intubation, intravenous cannulation, the administration of intravenous fluids, and the use of selected drugs (Calicott 1980). In high-income countries a substantial proportion of ambulance crews now include an ALS-trained officer. For example, in the UK, Department of Health policy requires that all emergency ambulances include an ambulance officer trained in ALS.

In response to the increasing global burden of injury, LMICs are rapidly adopting models of trauma care initially developed in highincome countries, such as ALS training for ambulance crews to improve outcomes in injury victims (Ali 1993; Sethi 2000). In many LMICs, the majority of patients arrive by private transport, although many countries have been developing pre-hospital care services further (Hauswald 1997; Areola-Risa 2000). As LMICs consider various models of pre-hospital care, the use of ALS training for ambulance crews has been debated (Sklar 1988; VanRooyen 1999). Little systematic evaluation of existing evidence is available for such policy-making.

\section{Why it is important to do this review}

The evidence for the impact of ALS-trained ambulance crews has yet to be rigorously appraised. The aim of this systematic review is therefore to quantify the impact of ambulance crews with ALS training on outcomes following trauma.

\section{O B J E C T I V E S}


To quantify the effectiveness of ALS-trained ambulance crews versus crews without ALS training on reducing mortality and morbidity in injured patients.

\section{METHODS}

\section{Criteria for considering studies for this review}

\section{Types of studies}

Randomised controlled trials (RCTs), quasi-randomised controlled trials (CCTs), before-and-after (CBAs, BAs) and interrupted time series (ITSs) studies.

\section{Types of participants}

All adult trauma patients over 18 years.

\section{Types of interventions}

ALS-trained ambulance crews versus crews without ALS training.

\section{Types of outcome measures}

- Death from all causes at the end of the follow-up period scheduled for each study

- Morbidity

\section{Search methods for identification of studies}

The searches were not restricted by date, language or publication status. Search terms were expanded for this version of the review to identify both randomised and non-randomised studies. Search strategies used for previous versions of the review can be obtained by contacting the Injuries Group Trials Search Co-ordinator.

\section{Electronic searches}

We searched the following electronic databases:

- Cochrane Injuries Group Specialised Register (searched July 8, 2009),

- CENTRAL (The Cochrane Library 2009, Issue 3),

- MEDLINE (Ovid SP) 1950 to June week 4 2009,

- EMBASE (Ovid SP) EMBASE 1980 to 2009 (Week 27),

- CINAHL (EBSCO) 1982 to June 2009,

- PubMed [www.ncbi.nlm.nih.gov/sites/entrez/] (searched July 82009 (added to PubMed in the last 90 days).

The search strategies are listed in full in Appendix 1.

\section{Searching other resources}

We screened the reference lists of all trial reports included in the review to identify any further published and unpublished data. We performed a general Internet search to identify grey literature.

\section{Data collection and analysis}

\section{Selection of studies}

One review author (SJ) examined the electronic search results, applied the selection criteria to the trial reports and retrieved these in full.

\section{Data extraction and management}

One review author (SJ) extracted information on the following: type of study design, stratification for effect modifiers, method of allocation concealment, number of randomised patients, type of participants, and interventions and outcomes. The outcome data sought were mortality and morbidity. The review author was not blinded to the authors or journal when doing this, although evidence for the value of blinding the review authors is not conclusive (Berlin 1997)

\section{Assessment of risk of bias in included studies}

We assessed study quality using the recommendations outlined by the Cochrane Handbook for Systematic Reviews of Interventions to determine the degree to which systematic bias may have been introduced, such as: bias through selection, performance, exclusion or detection; the method of allocation; the degree of followup, and the soundness of the assessments. One review author (SJ) categorised the studies as RCTs, CCTs, BAs and ITSs and applied these specific categories of quality assessment to the trial reports. Relevant reports were retrieved in full.

\section{Assessment of heterogeneity}

The groups of trials would have been examined for statistical evidence of heterogeneity using the $\mathrm{Chi}^{2}$ test. If there was no obvious heterogeneity on visual inspection or statistical testing, pooled relative risks and $95 \%$ confidence intervals would have been calculated using a fixed-effect model.

\section{Subgroup analysis and investigation of heterogeneity}

The following comparisons were planned:

Mortality and morbidity of victims of trauma treated by ALStrained ambulance crews versus crews without ALS training. 'A priori' sub-group analyses reported by studies were evaluated to 
look for statistically significant differences that were not apparent in the overall analyses.

The intended analysis was the calculation of relative risk of death and $95 \%$ confidence interval for each trial, such that a relative risk of more than one indicates a higher risk of death. We chose relative risk as it can be more readily applied to clinical situations.

\section{Sensitivity analysis}

The effect of excluding trials judged to be inadequate according to the Cochrane Handbook for Systematic Reviews of Interventions (Higgins 2008) criteria for quality would have been examined in a sensitivity analysis.

\section{R E S U L T S}

\section{Description of studies}

See: Characteristics of included studies; Characteristics of excluded studies.

\section{Results of the search}

The search strategy identified a total of 2886 references. The search used for this update was a new search (rather than an updated search), and was broader than the one used for the original version of the review. The search covered all years to July 2009 and used terms to increase the sensitivity to find randomised and non-randomised studies. Three studies met our inclusion criteria. These three studies compared outcomes of trauma patients treated by ambulance crews with ALS training compared to crews without ALS training.

\section{Included studies}

Arreola-Risa 2004

This was a controlled before-and-after study on mortality from injury in three Mexican cities (Monterrey, San Pedro and Santa Catarina). In San Pedro, ambulance staff received training in Basic Trauma Life Support, Advanced Cardiac Life Support, and a locally designed airway course. In Monterrey, ambulance staff received training in pre-hospital Trauma Life Support (a BTLS equivalent). In Santa Catarina, no additional training was provided (i.e. this was the control group). Before and after comparisons were made in Monterrey in 1994 and 1995, and in 2000 and 2001 for both San Pedro and Santa Catarina. Data were collected using self-reported ambulance-run sheets. The main treatment outcome was mortality from trauma. Secondary outcomes included effects of training on use of three specific skills and arrival time.
The number of trauma patients treated by ambulance personnel during the study period were 866 in Monterrey, 510 in San Pedro, and 504 in Santa Catarina. The injured patients were predominantly male and sustained mostly blunt injuries. In the follow-up period, patients in Monterrey (BLS group) received statistically greater numbers of all procedures for the three key management areas: airway management, fluid resuscitation and spinal immobilisation whereas patients in San Pedro (ALS group) received better management in some areas but not all. Management of these three areas were unchanged in Santa Catarina (the control group). However, despite these improvements in process indicators in Monterrey and to a lesser extent in San Pedro, the study demonstrates no statistically significant change in mortality in any of the three settings. When deaths on scene were excluded, there was a small trend towards lower mortality among injured patients in Monterrey (BLS group) but not in the other two settings.

Nicholl 1998

This RCT compared outcomes of victims of trauma treated by ambulance crews with ALS training to outcomes of those treated by crews without ALS training. Participants were trauma (road traffic accidents, falls, work/chemical/sport accidents, self-harm, assaults and drowning) patients of all ages. People with superficial injuries were excluded. Follow-up was six months after the original incident and was performed using the SF-36 questionnaire. Protocol compliance was poor. The authors did not recruit sufficient numbers because of practical difficulties. The mortality and morbidity data of the randomised group were added to the main non-randomised cohort in the original analysis. Therefore, specific analysis of the randomised patients cannot be performed for this review.

Stiell 2008

This was a large scale uncontrolled before-and-after study which took place across 17 cities in Canada. The study addressed the impact of ALS training for the healthcare personnel who help patients before they reach hospital on patient mortality and morbidity due to injury. The study population included injured patients over 16 years of age with an injury severity score greater than 12 . Participants were transported by land ambulance and were treated at one of the 13 leading trauma hospitals in Ontario province. Data on pre-hospital care were collected from ambulance call reports and the provincial dispatch centre. Individual eligible patients with major trauma seen during the basic life-support phase (36 months) and the advanced life-support phase (36 months) were enrolled. The study intervention consisted of standardised national curriculum on advanced life-support and clinical training period programs. A total of 400 paramedics were trained to perform endotracheal intubation, insert intravenous lines and administer medications and fluids intravenously. The primary outcome was survival to hospital discharge (alive or being transferred to a long-term care facility) and was obtained from hospital records. Additionally, disease-specific quality of life in survivors was measured with the 7-level functional independence measure at dis- 
charge and six months. The sample size was determined assuming a minimum absolute difference in the primary outcome of 3.8\% between study phases.

A total of 2867 patients were enrolled: 1373 in the BLS phase and 1494 in the ALS phase. There was no substantial difference in overall survival to hospital discharge by phase $(81.8 \%$ for BLS versus $81.1 \%$ for ALS). The only exception to this finding was a lower survival in an 'a priori' sub-group of cases with an initial Glasgow Coma Scale score of less than nine. This group had lower survival rates in the advanced life-support phase than in the basic life-support phase $(60.1 \%$ versus $51.2 \%$; $\mathrm{P}=0.03)$. There were no differences in morbidity between the phases, as indicated by the Glasgow Outcome Scale and functional independence measure at discharge and six months after discharge. Based on the revised trauma score obtained from the trauma hospital, mortality was non-significantly increased for patients in the ALS phase (adjusted odds ratio $=1.2$ ), but when the pre-hospital revised trauma score was used, mortality was significantly worse in the intervention phase (adjusted odds ratio $=1.4,95 \%$ CI 1.0 to 1.9 ). The presence of ALS providers at the scene and intubation in the field were associated with increased mortality (adjusted OR 1.5, 95\% CI 1.1 to 2.0 and adjusted OR 2.8, 95\% CI 1.6 to 5.0 , respectively). However, total response time was statistically longer in the ALS group than in the BLS group. Univariate analysis revealed that non-survivors were more likely to be intubated in the field than survivors, and intubations in the field were associated with increased odds of death in multivariate analysis. Based on this, ALS procedures were thought to lead to delays and longer response times which may explain why better trained paramedics have worse outcomes than others.

The characteristics of these studies are included in the Characteristics of included studies table.

\section{Excluded studies}

Messick 1992 published a descriptive study comparing counties within the state of North Carolina, USA that have ALS-trained versus BLS-trained emergency medical technicians (EMT). On multivariate analysis, counties with BLS-trained pre-hospital personnel were found to have higher death rates than counties with ALS-trained staff. However, because of the weak descriptive design, this study did not fit the previously established inclusion criteria and was excluded from this review.

Liberman 2003 conducted a prospective cohort study to evaluate three types of pre-hospital systems across three cities in Canada and their impact on injury mortality. Physicians trained using ALS to provide emergency care were compared to paramedics with ALS training and emergency medical technicians (EMT) with BLS training. The study demonstrated that overall injury mortality was lower in the patient group treated with BLS than that treated with ALS (18\% versus $29 \%$ ). There was a $35 \%$ mortality rate in the group receiving care from physicians, a $24 \%$ mortality rate in the group treated by a paramedic, and an $18 \%$ mortality rate in the group treated by an EMT. However, this study did not fit the previously established inclusion criteria based on design and was excluded from this review.

Eckstein 2000 reported the results of a prospective cohort study of injured patients who received either bag-valve-mask or endotracheal intubation for breathing assistance in the pre-hospital setting. After adjusting for the mechanism of injury and injury severity, patients who had received bag-valve-mask ventilation by paramedics were five times more likely to survive than those who were intubated. This study was not included in this review due to its study design, which was prone to bias.

Sampalis 1993 evaluated the effect of on-scene care, pre-hospital times and care at the hospital on injury mortality using a casecontrol design. Using multiple logistic regression they showed that on-scene ALS did not improve survival and that long pre-hospital times (> 60 minutes) were associated with an increased odds of dying (OR of 3.0). This study was not included in the review because its case-control design did not fit the previously established inclusion criteria.

Sukamaran 2005 conducted a cohort study comparing the care provided by paramedics with ALS training and technicians with BLS training and outcomes of injury victims treated by each group. They found no difference in mortality for patients treated by either group when adjusting for age, Glasgow Coma Score and injury severity. There was no significant difference between the two groups when patients with penetrating injuries were compared, although blunt trauma patients treated by paramedics had a higher mortality rate. Paramedics spent longer at the scene and had longer total pre-hospital times than technicians, they saw sicker patients and called for standby support more frequently. This study was not included in the review because it did not fit the previously established inclusion criteria based on study design.

\section{Risk of bias in included studies}

Arreola-Risa 2004: Limitations that may have biased the results include having only one control site, testing the intervention in only a few sites, difference in the time frame between groups, and a lack of sample size calculations to evaluate appropriate effect sizes. Because this was not a randomised controlled trial, allocation and blinding could not be done. Thus potential confounders may not have been adequately addressed.

Nicholl 1998: The dispatch of ambulance crews was randomised by opening a sealed, numbered envelope when a potential eligible emergency call was received by the dispatcher. Blinding of outcome assessments was not reported. The poor protocol compliance could have affected the results.

Stiell 2008: The most important limitation of this study was its design as an uncontrolled before-and-after study rather than a randomised trial, which is the gold standard to test such an intervention. The authors suggest that since randomised trials are challeng- 
ing to conduct in the pre-hospital setting for ethical reasons, the study design was, in this case, the optimal method of answering the study question. Furthermore, sub-group analyses suggested higher mortality in patients with a lower Glasgow Coma Score although this may be a false positive finding and would need to be evaluated further.

\section{Effects of interventions}

All three studies included in this review demonstrated no reduction in mortality for injured patients receiving aid from ALS-trained ambulance crews versus BLS-trained crews. The only randomised controlled trial was too small to show the impact of ALS training on injury mortality and morbidity. There may be some evidence to suggest that such training negatively affects mortality in some groups. In Stiell 2008, an 'a priori' sub-group analysis demonstrated an increase in mortality among patients in the intervention group who had a Glasgow Coma Score less than nine. Additionally, when the pre-hospital trauma score was taken into account in logistic regression analysis, mortality in the patients receiving care from ALS trained crews increased significantly.

\section{DISCUSSION}

Three studies met the inclusion criteria for this review. This is in spite of conducting a very thorough literature search in which 2886 citations were screened to identify eligible trials. We believe it is unlikely that relevant trials have been overlooked.

Based on the limited data, there is at present no evidence to recommend ALS training of ambulance crews to care for injury victims. This finding highlights the lack of evidence on which current practice and policy in many high-income countries is based, where pre-hospital care is often provided by ambulance crews with ALS training. It emphasises the need to conduct well-designed intervention studies to establish this effectiveness and inform policy making in trauma services.

The lack of rigorous research may not be easily rectified in settings where ALS-based services have already been established. There is conviction among the public, the media, and health professionals (including ambulance service staff), that ALS interventions are beneficial in serious trauma. However, despite the practical problems that may be experienced during research, randomised controlled trials remain the most rigorous research design for evaluating health care interventions.
A number of other factors need to be taken into account in planning an evaluative and comparative investigation in pre-hospital care of injury victims. These include the impact of ALS interventions on scene time, the impact of scene time on outcomes, the mechanism of trauma (blunt versus penetrating), geographical location (distance from hospital care), injury severity, injury pattern (presence and severity of head injury) and mode of pre-hospital transport. In addition, the configuration of pre-hospital services needs to be considered. For example, in some countries ambulances are staffed by doctors, many of whom have postgraduate or specialist training in intensive care or anaesthesia, which may affect outcomes. The model of pre-hospital services, therefore, may be a significant component in future studies and may limit comparability of studies.

\section{A U THORS' CONCLUSIONS}

\section{Implications for practice}

There is no evidence of the effectiveness of advanced life support training for ambulance crews on injury mortality or morbidity.

\section{Implications for research}

There is wide acceptance in high income countries that ALStrained ambulance crews are beneficial to major injury victims, spurring its widespread implementation. However, no rigorous evidence supports such policy at this time. A large randomised controlled trial would provide the most reliable evidence of effectiveness of this intervention. Additionally, the use of a step-wedge design could address some of the ethical concerns such as the perceived lack of equipoise between care by ALS-trained staff versus that of BLS-trained staff.

\section{ACKNOWLEDGEMENTS}

We thank Drs. D. Mohan, C. Mock, R. Norton and M. Varghese of the WHO Pre-hospital Trauma Care Steering Committee for their comments and advice on the review. We thank Mr. R. Wentz and Mrs. K. Blackhall for their help with conducting the searches.

To Frances Bunn, Ian Roberts, Anne-Maree Kelly, Pablo Perel and Irene Kwan, many thanks for their contribution to the protocol and previous versions of the review. 


\section{R E F E R E N C E S}

\section{References to studies included in this review}

Arreola-Risa 2004 \{published data only\}

Arreola-Risa C, Mock C, Herrera-Escamilla A, Contreras I, Vargas J. Cost-Effectiveness and Benefit of Alternatives to Improve Training for Prehospital TraumaCare in Mexico. Prehospital and Disaster Medicine 2004;19(4):318-25.

Nicholl 1998 \{published data only\}

Nicholl J, Hughes S, Dixon S, Turner J, Yates D. The costs and benefits of paramedic skills in pre-hospital trauma care. Health Technology Assessment 1998;2(17):no pp.

Stiell 2008 \{published data only\}

Stiell I, Nesbitt L, Pickett W, Munkley D, Spaite D, Banek J, et al for the OPALS Study Group. The OPALS Major Trauma Study: impact of advanced life-support on survival and morbidity. Canadian Medical Association Journal 2008;178(9):1141-52.

\section{References to studies excluded from this review}

Eckstein 2000 \{published data only\}

Eckstein M, Chan L, Schneir A, Palmer R. Effect of Prehospital Advanced Life Support on Outcomes of Major Trauma Patients. The Journal of Trauma: Injury, Infection, and Critical Care 2000;48 (4):643-8.

\section{Liberman 2003 \{published data only\}}

Liberman M, Mulder D, Lavoie A, Denis R, Sampalis J.

Multicenter Canadian Study of Prehospital Trauma Care. Annals of Surgery 2004;237(2):153-60.

Messick 1992 \{published data only\}

Messick W, Rutledge R, Meyer A. The Association of Advanced Life Support Training and Decreased Per Capita Trauma Death Rates: An Analysis of 12,417 Trauma Deaths. The Journal of Trauma 1992;33(6):850-5.

Sampalis 1993 \{published data only\}

Sampalis JS, Lavoie A, Williams JI, Mulder DS, Kalina M. Impact of On-site Care, Prehospital Time, and Level of In-hospital Care on Survival in Severely Injured Patients. The Journal of Trauma 1993; 34(2):252-61.

Sukamaran 2005 \{published data only\}

Sukumaran S, Henry JM, Beard D, Lawrenson R, Gordon MWG, O'Donnell JJ, et al.Prehospital trauma management: a national study of paramedic activities. Emergency Medicine Journal 2005;22: 60-3.

\section{Additional references}

\section{Ali 1993}

Ali J, Adam R, Butler AK, Chang H, Howard M, Gonsalves D, et al.Trauma outcome improves following the advanced trauma life support program in a developing country. The Journal of Trauma 1993;34:890-9.

Areola-Risa 2000

Areola-Risa C, Mock CN, Lojero-Wheatly L, de la Cruz O, Garcia C, Canavati-Ayub F, Jurkovich GJ. Low cost improvements in prehospital trauma care in a Latin American city. The Journal of Trauma 2000;48:119-24.

\section{Berger 1996}

Berger LR, Mohan D. Injury control: a global view. Oxford: Oxford University Press, 1996.

\section{Berlin 1997}

Berlin JA. Does blinding of readers affect the results of metaanalyses?. Lancet 1997;350:185-6.

\section{Cales 1984}

Cales RH. Trauma mortality in Orange County: The effects of implementation of a regional trauma system. Annals of Emergency Medicine 1984;13:1-8.

\section{Calicott 1980}

Calicott PE, Hughes I. Training in Trauma Advanced Life Support. Journal of the American Medical Association 1998;243:1156.

\section{Gwatkin 1997}

Gwatkin DR, Heuveline P. Improving the health of the world's poor. Communicable diseases among young people remain central. BMJ 1997;315:497.

\section{Hauswald 1997}

Hauswald M, Yeoh E. Designing a prehospital system for a developing country: estimated costs and benefits. American Journal of Emergency Medicine 1997;15:600-3.

\section{Higgins 2008}

Higgins JPT, Green S (editors). Cochrane Handbook for Systematic Reviews of Interventions Version 5.0.2 [updated September 2009]. The Cochrane Collaboration, 2008. Available from www.cochrane-handbook.org.

Kirsch 1998

Kirsch TD. Emergency medicine around the world. Annals of Emergency Medicine 1998;32:237-8.

\section{Lecky 2000}

Lecky F, Woodford M, Yates DW. Trends in trauma care in England and Wales 1989-97. Lancet 2000;355:1771-5.

\section{Lopez 2006}

Lopez AD, Mathers CD, Ezzati M, Jamison DT, Murray CJ. Global and regional burden of disease and risk factors, 2001: systematic analysis of population health data. The Lancet 2006;367 (9524):1747-57.

\section{Mathers 2006}

Mathers CD, Loncar D. Projections of global mortality and burden of disease from 2002 to 2030. PLoS Medicine 2006;3(11):e442.

Murray 1997a

Murray CJL, Lopez AD. Mortality by cause for the eight regions of the world: Global Burden of Disease Study. Lancet 1997;349: 1269-76.

\section{Murray 1997b}

Murray CJL, Lopez AD. Global mortality, disability, and the contribution of risk factors: Global Burden of Disease Study. Lancet 1997;349:1436-42.

Murray 1997c

Murray CJL, Lopez AD. Alternative projections of mortality and disability by cause 1990-2020: Global Burden of Disease Study. Lancet. 1997;349:1498-504. 


\section{Reines 1998}

Reines HD, Bartlett RL, Chudy NE, Kiragu KR, McKnew M. Is advanced life support appropriate for victims of motor vehicle accidents: the South Carolina highway trauma project. Journal of Trauma 1998;28:563-70.

\section{Roberts 1996}

Roberts I, Cambell F, Hollis SS, Yates D. Reducing accident death rates in children and young adults: the contribution of hospital care. BMJ 1996;313:1239-41.

\section{Sethi 2000}

Sethi D, Aljunid S, Sulong SB, Zwi A. Injury care in low-and middle-income countries: identifying potential for change. Injury Control \& Safety Promotion 2000;7:153-64.

\section{Sklar 1988}

Sklar DP. Emergency medicine and the developing world. American Journal of Emergency Medicine 1988;6:390-3

\section{VanRooyen 1999}

VanRooyen MJ, Thomas TL, Clem KJ. International emergency medical services: assessment of developing prehospital systems abroad. Journal of Emergency Medicine 1999;17:691-6.

* Indicates the major publication for the study 
CHARACTERISTICS OF STUDIES

Characteristics of included studies [ordered by study ID]

Arreola-Risa 2004

\begin{tabular}{|c|c|c|}
\hline Methods & \multicolumn{2}{|c|}{ Controlled before-and-after study. } \\
\hline Participants & \multicolumn{2}{|c|}{ Injured patients from three Mexican cities (Monterrey, San Pedro and Santa Catarina). } \\
\hline Interventions & \multicolumn{2}{|c|}{$\begin{array}{l}\text { In San Pedro: Basic Trauma Life Support plus Advanced Cardiac Life Support plus a } \\
\text { locally-designed airway course. } \\
\text { In Monterrey: pre-hospital Trauma Life Support (BTLS equivalent). } \\
\text { In Santa Catarina: no extra training (i.e. control group). }\end{array}$} \\
\hline Outcomes & \multicolumn{2}{|c|}{$\begin{array}{l}\text { Process indicators: use of procedures involving airway management, spinal immobilisa- } \\
\text { tion and fluid resuscitations. } \\
\text { Impact indicators: mortality in all injured patients treated by ambulance personnel during } \\
\text { the study period and mortality of all injured patients transported by ambulance personnel } \\
\text { during the study period (i.e. exclusive of field deaths). }\end{array}$} \\
\hline Notes & \multicolumn{2}{|c|}{$\begin{array}{l}\text { Before and after comparisons were made in } 1994 \text { and 1995, respectively, for Monterrey, } \\
\text { and } 2000 \text { and 2001, respectively, for both San Pedro and Santa Catarina. Data was } \\
\text { collected by self-report on ambulance run-sheets. No sample size calculations were noted. }\end{array}$} \\
\hline \multicolumn{3}{|l|}{ Risk of bias } \\
\hline Item & Authors' judgement & Description \\
\hline $\begin{array}{l}\text { Incomplete outcome data addressed? } \\
\text { All outcomes }\end{array}$ & Unclear & $\begin{array}{l}\text { It is unclear how many ambulance crews or per- } \\
\text { sonnel did not participate in the study or if there } \\
\text { was attrition of trained crew members during the } \\
\text { study time period and if this influenced outcomes } \\
\text { in any way. Authors also do not report if mortality } \\
\text { data was not collected or missing for any eligible } \\
\text { patients who were entered in the study. }\end{array}$ \\
\hline Free of selective reporting? & No & $\begin{array}{l}\text { Deaths in one city were assessed differently com- } \\
\text { pared to those in the other two cities. In Monterrey, } \\
\text { deaths in the field or en route to the Emergency } \\
\text { Department were captured, but not those that oc- } \\
\text { curred later in the Emergency Department stay or } \\
\text { in the hospital. In San Pedro and Santa Catarina, } \\
\text { hospital records were used to ascertain deaths, es- } \\
\text { pecially for patients at high risk of mortality. }\end{array}$ \\
\hline Free of other bias? & Unclear & $\begin{array}{l}\text { Authors do not report if the ambulance crews in the } \\
\text { cities were aware of what interventions were being } \\
\text { provided by the other. It appears that no blinding }\end{array}$ \\
\hline
\end{tabular}

Advanced trauma life support training for ambulance crews (Review) 
Arreola-Risa 2004 (Continued)

of this information occurred.

\section{Nicholl 1998}

Methods

Randomised controlled trial (of dispatch of paramedics and technicians by opening sealed numbered envelopes when a potentially eligible emergency call was received).

Decisions about whether to include a patient were made after randomisation, according to whether the inclusion criteria was met.

Participants

16 trauma patients of all ages, hospitalised due to road traffic accidents, falls, work/chemical/sport accidents, self-harm and drowning.

Inclusion and exclusion criteria retrospectively applied:

Included:

Length of hospital 1 . stay $=/>3$ days,

2. Admissions to ICU/HDU,

Deaths between ambulance arrival on scene and arrival at hospital.

3.

Transfer to another hospital or hospital's ICU/HDU with stay $=1>3$ days,

4.

5. Re-admission within 2 days of the incident,

6. All deaths within 6 months of the incident.

Excluded:

1. Poisonings,

2. Transported by helicopter,

3. Attended by doctors on scene,

4. Deaths before ambulance arrival,

5. Superficial skin injuries and burns,

6. Simple fracture of femur in patients $>65$ years old,

7. Simple spinal strain with no fracture,

8. Patients involved in 'major incidents'.

Interventions

Pre-hospital trauma care provided by ALS trained paramedic $(\mathrm{n}=8)$.

Pre-hospital trauma care provided by BLS trained

emergency technicians $(\mathrm{n}=8)$.

Outcomes

1. process of care,

2. morbidity as measured by general health perception and quality of life in a 6-month follow-up postal questionnaire (SF-36),

3. death within 6 months of the incident.

Notes

Poor protocol compliance.

Mortality and morbidity data of these 16 cases were added to main non-randomised cohort for analysis. Author contacted and data will be available in due course.

\section{Risk of bias}


Nicholl 1998 (Continued)

\begin{tabular}{l|ll}
\hline Item & Authors' judgement & Description \\
\hline Allocation concealment? & Yes & Adequate -- sealed numbered envelopes. \\
\hline $\begin{array}{l}\text { Incomplete outcome data addressed? } \\
\text { All outcomes }\end{array}$ & Unclear & - \\
\hline Free of selective reporting? & Unclear & - \\
\hline Free of other bias? & Unclear & - \\
\hline
\end{tabular}

\section{Stiell 2008}

\begin{tabular}{ll}
\hline Methods & Uncontrolled before-and-after study. \\
\hline Participants & $\begin{array}{l}\text { Injured patients over } 16 \text { years of age with an injury severity score greater than 12, all } \\
\text { of whom were transported by land ambulance and treated at one of the } 13 \text { lead trauma } \\
\text { hospitals in Ontario province. }\end{array}$ \\
\hline Interventions & $\begin{array}{l}\text { Two phases: basic life-support and then advanced life-support training and interventions } \\
\text { were conducted, each } 36 \text { months in duration. ALS training consisted of a standardised } \\
\text { national curriculum on advanced life-support and clinical training programs. Training } \\
\text { included use of endotracheal intubation, intravenous line placement and use of intra- } \\
\text { venous medications and fluids. }\end{array}$ \\
\hline
\end{tabular}

Outcomes

The primary outcome was survival to hospital discharge (alive, or being transferred to a long-term care facility) and was obtained from hospital records. Additionally, diseasespecific quality of life in survivors was measured with the 7-level functional independence measure at discharge and six months.

Non-randomised. No contemporaneous controls.

\section{Risk of bias}

\begin{tabular}{ll|l}
\hline Item & Authors' judgement & Description \\
\hline $\begin{array}{l}\text { Incomplete outcome data addressed? } \\
\text { All outcomes }\end{array}$ & Unclear & $\begin{array}{l}\text { Authors do not report attrition in ambulance } \\
\text { personnel during the study time period and if } \\
\text { this influenced outcomes in any way. }\end{array}$ \\
\hline Free of selective reporting? & Yes & $\begin{array}{l}\text { Outcomes were collected from hospital records } \\
\text { from all settings and analyses take into account } \\
\text { missing data or patients who became lost to fol- } \\
\text { low-up. }\end{array}$ \\
\hline Free of other bias? & Yes & $\begin{array}{l}\text { Authors conducted and reported a detailed anal- } \\
\text { ysis of potential confounders and other explana- }\end{array}$ \\
\hline
\end{tabular}


Stiell 2008 (Continued)

tions for the study's results although the study design increases risk of bias.

\section{Characteristics of excluded studies [ordered by study ID]}

Eckstein 2000 A prospective cohort study of injured patients who received either bag-valve-mask or endotracheal intubation for breathing assistance in the pre-hospital setting, did not fit the previously established study design criteria.

Liberman 2003 A prospective cohort study, did not fit the previously established study design criteria.

Messick 1992 A descriptive study, did not fit the previously established study design criteria.

Sampalis 1993 A case-control study, did not fit previously established inclusion criteria.

Sukamaran 2005 A cohort study comparing the care provided by paramedics with ALS training and technicians with BLS training and outcomes of injury victims treated by each group. Did not fit the previously established inclusion criteria. 


\section{DATA AND ANALYSES}

This review has no analyses.

\section{A P P E N D I C E S}

\section{Appendix I. Search strategy}

\section{Cochrane Injuries Group Specialised Register (searched July 8, 2009)}

((emerg* or trauma) and (prehospital or pre-hospital or preclinical or pre-clinical)) or "life support" or "Primary survey" or "golden hour" or "first aid" or "early management" or EMST or "advanced trauma life support" or ATLS or "advanced life support" or ALS or ("basic life support") or BLS

\section{CENTRAL (The Cochrane Library 2008, Issue 3)}

\#1MeSH descriptor Emergency Medical Services explode all trees \#2MeSH descriptor Critical Care explode all trees \#3MeSH descriptor Emergency Treatment explode all trees \#4MeSH descriptor Resuscitation explode all trees \#5MeSH descriptor Emergency Medicine explode all trees \#6MeSH descriptor First Aid explode all trees $\# 7 \mathrm{MeSH}$ descriptor Traumatology explode all trees \#8(\#1 OR \#2 OR \#3 OR \#4 OR \#5 OR \#6 OR \#7) \#9MeSH descriptor Advanced Cardiac Life Support explode all trees \#10(“Advanced trauma life support" or ATLS) NOT (ATLS near3 syndrome*) \#11(Advanced life support) or ALS \#12(basic life support) or BLS \#13(emergency or trauma or critical) near3 (care or treat ${ }^{*}$ )

\#14(trauma near3 system*) or (life near3 support*) or (primary near3 survey*) or (golden near3 hour) or (first near3 aid*) \#15(early management near3 trauma) or EMST

\#16(prehospital or pre-hospital or preclinical or pre-clinical) near3 (care or support or treat*)

\#17(\#9 OR \#10 OR \#11 OR \#12 OR \#13 OR \#14 OR \#15 OR \#16)

\#18MeSH descriptor Health Personnel explode all trees

\#19MeSH descriptor Allied Health Personnel explode all trees

\#20MeSH descriptor Medical Staff explode all trees

\#21MeSH descriptor Emergency Medical Technicians explode all trees

\#22paramedic*

$\# 23$ (emergency or critical or trauma or triage or ambulanc*) near3 (doctor* or crew* or staff or team*)

\#24(\#18 OR \#19 OR \#20 OR \#21 OR \#22 OR \#23)

\#25(\#8 AND \#17 AND \#24)

\section{MEDLINE (Ovid) 1950 to June Week 4 2009:}

1.exp Emergency Medical Services/

2.exp Critical Care/

3.exp Emergency Treatment/

4.exp Resuscitation/

5.exp Emergency Medicine/

6.exp First Aid/

7.exp Traumatology/

8.1 or 2 or 3 or 4 or 5 or 6 or 7

Advanced trauma life support training for ambulance crews (Review) 
9.exp Advanced cardiac life support/

10.((Advanced trauma life support or ATLS) not (ATLS adj3 syndrome*)).ti,ab.

11.(Advanced life support or ALS).ti,ab.

12.(basic life support or BLS).ab,ti.

13. ((emergency or trauma or critical) adj3 (care or treat*)).ab,ti.

14.((trauma adj3 system*) or (life adj3 support*) or (primary adj3 survey*) or (golden adj3 hour) or (first adj3 aid*)).ab,ti.

15.EMST.ab,ti.

16.(early management adj3 trauma).ab,ti.

17.((prehospital or pre-hospital or preclinical or pre-clinical) adj3 (care or support or treat*)).ab,ti.

18.9 or 10 or 11 or 12 or 13 or 14 or 15 or 16 or 17

19.8 and 18

20.exp health personnel/

21.exp allied health personnel/

22.Medical staff/

23.paramedic*.ab,ti.

24.exp Emergency Medical Technicians/

25.((emergency or critical or trauma or triage or ambulanc $\left.{ }^{*}\right)$ adj3 (doctor* or crew* or staff or team*)).ab,ti.

26.20 or 21 or 22 or 23 or 24 or 25

27.19 and 26

28.clinical trials as topic.sh.

29.randomi?ed.ti,ab.

30.randomized controlled trial.pt.

31.controlled clinical trial.pt.

32. (controlled adj3 ("before and after" or trial* or study or studies or evaluat*)).ab,ti.

33.randomized.ab.

34.placebo.ti,ab.

35.((before adj3 after) or (interrupted adj3 time)).ab,ti.

36.randomly.ab.

37.trial.ti.

38. (groups or cohorts).ti,ab.

39. (observed or observation*).mp.

40.((compar* or intervention or evaluat*) adj3 (trial* or stud*)).ab,ti.

41.(random* adj3 allocat*).ab,ti.

42.exp prospective studies/

43.exp follow-up studies/

44.exp comparative study/

45.exp cohort studies/

46.exp evaluation studies/

47.28 or 29 or 30 or 31 or 32 or 33 or 34 or 35 or 36 or 37 or 38 or 39 or 40 or 41 or 42 or 43 or 44 or 45 or 46

48.27 and 47

\section{EMBASE 1980 to 2009 Week 27}

1.exp Emergency/

2.exp emergency health service/

3.exp Emergency Treatment/

4.exp intensive care/

5.exp resuscitation/

6.exp emergency medicine/

7.exp traumatology/

8.exp neurotraumatology/

9.exp First aid/

10.1 or 2 or 3 or 4 or 5 or 6 or 7 or 8 or 9

11.((Advanced trauma life support or ATLS) not (ATLS adj3 syndrome*)).ti,ab.

Advanced trauma life support training for ambulance crews (Review)

Copyright $(2010$ The Cochrane Collaboration. Published by John Wiley \& Sons, Ltd. 
12.(Advanced life support or ALS).ti,ab.

13.(basic life support or BLS).ab,ti.

14.((emergency or trauma or critical) adj3 (care or treat*)).ab,ti.

15.((trauma adj3 system*) or (life adj3 support*) or (primary adj3 survey*) or (golden adj3 hour) or (first adj3 aid*)).ab,ti.

16.EMST.ab,ti.

17.(early management adj3 trauma).ab,ti.

18.((prehospital or pre-hospital or preclinical or pre-clinical) adj3 (care or support or treat*)).ab,ti.

19.11 or 12 or 13 or 14 or 15 or 16 or 17 or 18

20.exp medical staff/

21.exp paramedical personnel/

22.paramedic*.ab,ti.

23. ((emergency or critical or trauma or triage or ambulanc*) adj3 (doctor* or crew* or staff or team*)).ab,ti.

24.20 or 21 or 22 or 23

25.10 and 19 and 24

26.exp Randomized Controlled Trial/

27.exp controlled clinical trial/

28.randomi?ed.ab.

29.placebo.ab.

30.exp Clinical Trial/

31.randomly.ab.

32.(random* adj3 allocat*).ab,ti.

33.trial.ti.

34. (controlled adj3 ("before and after" or trial* or study or studies or evaluat*)).ab,ti.

35.((before adj3 after) or (interrupted adj3 time)).ab,ti.

36. (groups or cohorts).ab,ti.

37.(observ* adj3 (trial* or stud*)).ab,ti.

38.((compar* ${ }^{*}$ or intervention* ${ }^{*}$ or evaluat $\left.{ }^{*}\right)$ adj3 (trial* or stud $\left.\left.^{*}\right)\right)$.ab,ti.

39.exp prospective study/

40.exp follow up/

41.exp comparative study/

42.exp experimental study/

43.observational study/

44.exp quasi experimental study/

45.exp cohort analysis/

46.exp evaluation research/

47.exp time series analysis/

48.or/26-47

49.25 and 48

CINAHL (EBSCOHOST) 1937 to June 2009

S1 TX (Advanced trauma life support or ATLS) NOT (ATLS W3 syndrome)

S2 TX Advanced life support or ALS

S3 TX basic life support or BLS

S4 TI ( (emergency or trauma or critical) ) and TI ( (care or treat*) ) or AB ( (emergency or trauma or critical) ) and AB ( (care or treat*)

S5 AB ( (trauma AND system*) or (life AND support*) or (primary AND survey*) or (golden AND hour) or (first AND aid*) ) or TI ( (trauma AND system*) or (life AND support*) or (primary AND survey*) or (golden AND hour) or (first AND aid*)

S6 TX (early management and trauma) OR (EMST)

S7 AB ( (prehospital or pre-hospital or preclinical or pre-clinical) AND (care or support or treat or treatment*) ) or TI ( (prehospital or pre-hospital or preclinical or pre-clinical) AND (care or support or treat or treatment*) )

S8 $\mathrm{S} 1$ or $\mathrm{S} 2$ or S3 or $\mathrm{S} 4$ or $\mathrm{S} 5$ or S6 or S7

S9(MH "Health Personnel+") or (MH "Allied Health Personnel+") or (MH "Emergency Medical Technicians")

S10paramedic*

S11(emergency or critical or trauma or triage or ambulanc*) and (doctor* or crew* or staff or team*)

Advanced trauma life support training for ambulance crews (Review) 
S12S9 or $\mathrm{S} 10$ or $\mathrm{S} 11$

$\mathrm{S} 13 \mathrm{~S} 8$ and $\mathrm{S} 12$

S14 Non-RCT search strategy saved to server

S15 S13 and S14

PubMed [www.ncbi.nlm.nih.gov/sites/entrez/] (searched July 92009 (added to PubMed in the last 90 days)

\#1Search (((("Emergency Medical Services"[Mesh] OR "Critical Care“[Mesh]) OR ”Emergency Treatment"[Mesh]) OR ”Resuscitation“"[Mesh]) OR ”Emergency Medicine“[Mesh]) OR ”First Aid“[Mesh]) OR ”Traumatology“[Mesh]

\#2 Search "Advanced Cardiac Life Support“[Mesh]

\#3 Search ("Advanced trauma life support" OR ATLS) NOT (ATLS AND syndrome*) Field: Title/Abstract

\#4 Search Advanced life support or ALS Field: Title/Abstract

\#5 Search basic life support OR BLS Field: Title/Abstract

\#6 Search (emergency or trauma or critical) AND (care or treat*) Field: Title/Abstract

\#7 Search (emergency or trauma or critical) AND (care or treatment*) Field: Title/Abstract

\#8 Search (trauma AND system) or (life AND support*) or (primary AND survey*) or (golden AND hour) or (first AND aid*) Field:

Title/Abstract

\#9 Search (early management AND trauma) OR EMST Field: Title/Abstract

\#10 Search (prehospital or pre-hospital or preclinical or pre-clinical) AND (care or support or treatment*) Field: Title/Abstract

\#11 \#2 OR \#3 OR \#4 OR \#5 OR \#6 OR \#7 O \#8 OR \#9 OR \#10

\#12 Search ((("Health Personnel“[Mesh] OR ”Allied Health Personnel“[Mesh])) OR ”Medical Staff“"[Mesh]) OR ”Emergency Medical

Technicians"[Mesh]

\#13 Search paramedic* or para-medic*

\#14 Search (emergency or critical or trauma or triage or ambulanc*) AND (doctor* or crew* or staff or team*)

\#15 \#12 OR \#13 OR \#14

\#16 \#1 AND \#11 AND \#15

\section{WHAT'S NEW}

Last assessed as up-to-date: 7 July 2009.

24 July 2009 New citation required and conclusions have changed

\section{H IS T O R Y}

Protocol first published: Issue 2, 2001

Review first published: Issue 2, 2001
Results from three new studies were included in this update. Conclusions changed from absence of evidence of effectiveness to no evidence to suggest a benefit from this intervention.

The title of the review has changed. The authors of the review have changed. The inclusion criteria of the review has been broadened and now includes interrupted time series studies. 


\section{CONTRIBUTIONSOFAUTHORS}

For the protocol, and review versions through the 2008 update: DS helped to design the protocol, examined search results, applied inclusion criteria and wrote the review. IK helped design the protocol, examined search results, applied inclusion criteria, obtained papers, extracted data, contacted authors and helped to write the review. AMK applied inclusion criteria, extracted data and helped to write the review. IR and FB commented on the protocol and helped to write the review.

For the 2009 update: SJ examined search results, obtained papers, applied inclusion criteria, extracted data, contacted authors and edited the review.

\section{DECLARATIONS OF INTEREST}

None known.

\section{SOURCES OFSUPPORT}

\section{Internal sources}

- Institute of Child Health, University of London, UK.

\section{External sources}

- Global Programme on Evidence of Health Policy (GPE), World Health Organization, Switzerland.

- University of California San Francisco, Department of Surgery, USA.

\section{DIFFERENCES BETWEEN PROTOCOLANDREVIEW}

The protocol was modified to include interrupted time series studies since this design is often used to test the effectiveness and impact of pre-hospital interventions based on the ethical considerations influencing any study of pre-hospital care.

\section{NDEX TERMS}




\section{Medical Subject Headings (MeSH)}

*Life Support Care; Ambulances; Controlled Clinical Trials as Topic; Emergency Medical Technicians [*education]; Randomized Controlled Trials as Topic; Traumatology [*education]

\section{MeSH check words}

Humans 